not time spent per COI (OR 0.89, 95\% [0.68 to 1.16], $\mathrm{p}=0.38, \mathrm{n}=11$ ). An ordinal regression model did not show an association between presenter's $\mathrm{H}$-index and total quality score (OR $0.94,95 \%$ CI [0.87 to 1.01$], \mathrm{p}=0.11, \mathrm{n}=11$ )

Conclusion A large minority of presenters did not display a COI statement. Those COI statements that were present were of variable quality. Many were displayed for insufficient time, lacked any verbal explanation or used a small font. These factors may limit the ability of audience members to understand the potential conflicts. Seniority of presenter correlated with number of conflicts but not quality of the COI statement. Strategies should be implemented to ensure all presenters outline whether they have any potential COI in a standardised manner.

Conflict of Interest None

\section{ANTIHYPERTENSIVE DRUG CONCORDANCE IN PATIENTS WITH APPARENT RESISTANT HYPERTENSION: A TERTIARY HYPERTENSION REFERRAL CENTRE EXPERIENCE}

Tarek Antonios*. St George's, University of London, London

10.1136/heartjnl-2019-BCS.113

Introduction Individuals with resistant hypertension have a $50 \%$ greater risk of cardiovascular events (death, myocardial infarction, heart failure, stroke or chronic kidney disease) compared to those with well-controlled hypertension. Nonadherence to antihypertensive medication is a major cause of uncontrolled hypertension, with rates reported as high as $84 \%$. The aim of this study was to assess the prevalence of non-adherence in hypertensive individuals referred to a tertiary hypertension centre in South of London (Blood Pressure Unit, St George's University Hospital NHS foundation Trust).

Methods After the optimisation of drug regimens and the exclusion of white coat and secondary hypertension, drug adherence was assessed using liquid chromatography-mass spectrometry (LC-MS) analysis of urine samples in 124 individuals who met the criteria for resistant hypertension (uncontrolled hypertension despite concurrently taking at least 3 antihypertensive drugs of different classes including a diuretic or hypertension controlled on 4 drugs). All included subjects confirmed adherence to their prescribed antihypertensive medication on several occasions, whilst individuals who admitted to missing their drugs were excluded from the study.

Results Non-adherence was found in 71 individuals (57.3\%). Of these $45(63.4 \%)$ had partial non-adherence and 26 $(36.6 \%)$ had complete non-adherence. Non-adherent individuals were significantly younger in age, had a shorter history of hypertension, more likely to be a female (OR: 3.07; 95\% CI: 1.45-6.47; $\mathrm{p}=0.003$ ) and have depression (OR: 11.66; 95\% CI 1.47-92.19; $\mathrm{p}=0.004)$, and less likely to have concomitant cardiovascular disease (OR: $0.42 \quad 95 \%$ CI $0.19-0.92$; $\mathrm{p}=0.028$ ), type 2 diabetes mellitus (OR: 0.40 ; 95\% CI: $0.19-$ $0.83 ; \mathrm{p}=0.013$ ), chronic kidney disease (OR: 0.34; 95\% CI $0.12-0.92 ; \mathrm{p}=0.028$ ) and retinopathy (OR: $0.14 ; 95 \% \mathrm{CI}$ $0.04-0.51 ; \mathrm{p}=0.001)$. Low levels of adherence were observed more for individuals on diuretics.

Conclusion In individuals with apparent resistant hypertension, poor adherence to antihypertensive therapy is very common and was observed in $57 \%$ of individuals despite their insistence of their treatment adherence in the preceding days. The assessment of adherence at an early stage in individuals with apparent resistant hypertension is essential for tailoring treatment strategies and for saving vital healthcare resources.

Conflict of Interest None

\section{CAN NON-MODIFIABLE RISK FACTORS OF AGE AND SEX BE USED TO DETERMINE WHICH PATIENTS WITH CHEST PAIN WE INVESTIGATE WITH CT CORONARY ANGIOGRAPHY (CTCA) AS AN INITIAL TEST}

${ }^{1}$ Ali Rauf*, ${ }^{2}$ Shazia Arshad, ${ }^{3}$ Sarah Denny, ${ }^{3}$ Floyd Pierres, ${ }^{3}$ Nikolaos Papamichail, ${ }^{4}$ Antonis Pavlidis, ${ }^{3}$ Khaled Alfakih. ' University College London; ${ }^{2}$ The Grove Medical Practice; ${ }^{3}$ Lewisham and Greenwich NHS Trust; ${ }^{4}$ Guy's and St. Thomas' NHS Foundation Trust

\subsection{6/heartjnl-2019-BCS.114}

Introduction The UK NICE guidelines for the investigation of chest pain were updated in 2016 to recommend CTCA as the initial test for all patients with stable chest pain. This was in comparison to the 2010 guidelines on stable chest pain which recommended that patients with mild and moderate pre-test probability (PTP) should be investigated non-invasively with cardiac CT and functional imaging tests respectively whilst patients with high PTP should be referred directly to invasive coronary angiography (ICA). The removal of PTP in the updated guidelines was in view of it overestimating prevalence of coronary artery disease (CAD).

Purpose We wanted to assess whether non-modifiable risk factors of age and sex can be used to better predict which patients with new onset chest pain should be investigated by a non-invasive test such as CTCA and whether there is still a role for direct referral for ICA

Method We examined our catheter lab database for patients undergoing ICA over a 12 month period for either new onset stable chest pain or acute coronary syndrome (ACS) who had not undergone any initial non-invasive test. We documented age and sex for all patients, and calculated percentage yield of severe CAD in each of the subgroups.

Results In total, 330 patients with stable chest pain and 250 with ACS were identified. The prevalence of severe CAD in these cohorts, grouped according to age and sex, are shown in table 1. Numbers of females under the age of 55 were lowest as might be expected, and this also corresponded with low \% yield of severe CAD in both the stable (14\%) and ACS (21\%) group. In the stable group, prevalence of severe $\mathrm{CAD}$ was relatively low in all other groups, and highest in males aged over 55 at $40 \%$. Percentage yield was above $50 \%$ in all males with ACS, and all females above the age of 55 .

Conclusion Our data demonstrates that in the patients with stable symptoms, who were referred directly for ICA and therefore had presumed high PTP, there was low prevalence of severe CAD particularly in young females. This would support NICE guidance to investigate these patients non-invasively. There may still be a role for investigating older men with ICA given the relatively higher prevalence. In patients presenting with ACS, our data suggests that there may also be a role for investigating young women by non-invasive means in the first instance.

Conflict of Interest None 